\title{
Cooperación e intercambio de conocimiento en redes inter-organizativas informales ${ }^{1}$
}

\author{
Carlos Martín-Ríos \\ Center for Organizational Learning \& Transformation. Rutgers, the State \\ University of New Brunswick. Nueva Jersey. Estados Unidos \\ Center for Management Innovation. Madrid. España. ${ }^{2}$ \\ Manuel Septiem
}

Sección de Organización de Empresas. Universidad Carlos III de Madrid.

España.

\section{Resumen}

El presente artículo explora el papel que juegan las relaciones informales de colaboración entre empresas en la existencia y sostenimiento de redes de intercambio de múltiples formas de conocimiento. Para ello, se reportan los resultados de un estudio empírico llevado a cabo en un colectivo de empresas localizadas en un campus tecnológico que constituyen una red completa interorganizacional. El estudio presenta evidencia sobre importancia de las características estructurales y las relaciones de confianza en el desarrollo de relaciones de colaboración para el intercambio de conocimiento en áreas relacionadas con la tecnología, el mercado y productos, y la gestión empresarial.

Palabras clave: Intercambio de conocimiento - Redes inter-organizacionales Relaciones informales - Reciprocidad - Análisis de redes sociales.

\begin{abstract}
This paper explores the role of informal and collaborative inter-firm relationships in the creation and promotion of networks of various sources of knowledge. It reports on an empirical research carried out on a sample of firms located in technology park. Firms form a complete inter-organizational network. This study illustrates the importance of structural characteristics and trust relationships in the development of collaborative relationships, which facilitate exchanging technological, market/product and organizational/managerial knowledge.
\end{abstract}

Key words: Knowledge exchange - Inter-firm networks - Informal relations Reciprocity - Social network analysis.

\footnotetext{
${ }^{1}$ Los autores agradecen al Parque Científico de la Universidad Carlos III de Madrid la colaboración y los medios prestados para garantizar las entrevistas con los directivos del parque. Agradecen especialmente a Lourdes Negro por su asistencia en diversas fases del proyecto. El presente estudio ha contado con la financiación del Ministerio de Educación y Ciencia, proyecto ECO2008-01513 y de la Comunidad de Madrid a través del Plan de I+D, proyecto PROLIPAPELII PROLIPAPEL 2010/0084/001.

2 Enviar correspondencia a: cmartinrios@cminnovation.org
} 
Para que las empresas puedan tener éxito en el contexto económico abierto y competitivo actual no es suficiente con canalizar y administrar recursos internos con los que desarrollar productos y servicios. Con frecuencia, las empresas se enfrentan a la necesidad de colaborar con otras empresas, incluso competidoras, para obtener información sobre aspectos esenciales como: la forma de organizar el trabajo, de innovar, de producir u ofrecer servicios. Las empresas necesitan, en definitiva, aprender unas de otras. Por ello, la habilidad de intercambiar conocimiento y la capacidad de aprendizaje desde una óptica cooperativa se está convirtiendo en un recurso crucial para ellas (Powell y col., 1996; Teece, 1987; Uzzi, 1997; Van Wijk y col., 2008). Diversos estudios muestran como las empresas establecen y participan en múltiples formas de relaciones e interacciones: de mercado o formales (por ejemplo las alianzas estratégicas y los acuerdos universidad-empresa) y colaborativas e informales, todas ellas con el fin de asegurarse el acceso al conocimiento en una variedad de ámbitos (Appleyard, 1996; Hagedoorn, 1993; Uzzi, 1997).

A pesar de lo atractivo del argumento expuesto, gran parte de la literatura académica se ha centrado en desentrañar el papel de las redes formales en la creación e intercambio de conocimiento formalmente organizado en un cuerpo de conocimiento específico. En concreto, buena parte de los estudios publicados sobre intercambio de conocimiento hacen referencia, casi en exclusividad, al conocimiento sobre cuestiones relativas a la tecnología en un sentido amplio que incluye la innovación, producción y comercialización, entre otros. El número de estudios que analizan el contexto, las características estructurales y las relaciones de confianza en el desarrollo de relaciones de colaboración para el intercambio de conocimiento en diversas áreas de conocimiento (Powel y col., 1996) es mucho menor. Si bien esta perspectiva puede ayudar a elaborar una imagen más detallada de las relaciones entre empresas, la idea de la heterogeneidad en el intercambio de varias formas de conocimiento aún no ha sido tenida en suficiente consideración.

Hasta fecha reciente no se han analizado en profundidad las implicaciones que las relaciones sociales (no contractuales) tienen en la obtención de conocimiento relevante para las empresas (Colombo y col., 2011; Tödtling y col., 2009). El presente estudio se enfoca en la existencia de relaciones colaborativas, informales entre empresas, para estudiar la naturaleza de las redes de intercambio de conocimiento entre empresas. En concreto, se plantean las siguientes preguntas de investigación: ¿hasta qué punto la participación en redes informales facilita que las empresas obtengan conocimiento en una variedad de dominios? De ser así ¿cuáles son las propiedades estructurales y relacionales de estas redes de conocimiento? 
En el presente estudio se analizarán aquellos factores estructurales y relacionales que la literatura ha mostrado son los más relevantes en lo que respecta al intercambio de conocimiento informal: centralización, densidad y reciprocidad (Easterby-Smith y col., 2008). A través del análisis de redes sociales, este artículo analiza empíricamente el intercambio de diversas formas de conocimiento en un colectivo de 50 empresas que conforman un parque científico y tecnológico, bajo la gestión de una universidad pública. Esta red de empresas ofrece la oportunidad de analizar el desarrollo de interacciones colaborativas, al tiempo que permite establecer una red cuyos límites están claramente delimitados.

El artículo está organizado en las siguientes secciones. A continuación, se describe el marco teórico relevante para este estudio. En la siguiente sección se explica el método de recogida de información y la metodología de análisis utilizada. En la sección de resultados se discuten las características de las redes informales de intercambio de conocimiento entre empresas del parque tecnológico. Este análisis sirve de base para extraer conclusiones acerca de las motivaciones para la creación de redes informales inter-organizacionales. Asimismo, se detallan las limitaciones del estudio y las líneas futuras de investigación.

\section{Las redes inter-organizacionales de intercambio de conocimiento}

Uno de los escenarios más apropiados para la adquisición e intercambio de conocimiento es a través de la red social, definida como el patrón de comunicación, amistad, asesoramiento o apoyo que existe entre los miembros de un sistema social (Burt y Minor, 1983). Las redes sociales, sus características y estructura son cruciales para determinar los flujos de intercambio de ideas, innovaciones o experiencias (Burt y Minor 1983; Scott 1991; Wellman 1983). Al utilizar el concepto de red social hacemos referencia a la teoría de redes sociales según la cual las empresas se encuentran conectadas unas con otras formando canales de comunicación e influencia (Granovetter, 1985). De acuerdo con la teoría de redes sociales, tanto la imbricación (o embeddedness) estructural como la relacional determinan la estructura de la red y la forma en que la transferencia de conocimiento va a tener lugar (Uzzi, 1997).

En general, los intercambios inter-organizacionales tienen lugar en dos escenarios diferenciados (Granovetter, 1992; Uzzi, 1997). Por un lado, el proceso de transferencia de conocimiento puede ser canalizado formalmente, de forma explícita, mediante el desarrollo de relaciones contractuales con otras empresas y organizaciones como, por ejemplo, en el caso de los contratos universidad-empresa 
(Grandori y Sosa, 1995; Liebeskind y col., 1996). Por otro lado, una buena parte del conocimiento, particularmente aquel con una dimensión tácita, a menudo se transfiere de manera informal a través de procesos de socialización e interiorización que tienen lugar en el marco de redes personales e informales (Powell y col., 1996).

El análisis de las redes informales o colaborativas de intercambio de conocimiento entre empresas-definidas como aquellos acuerdos voluntarios entre empresas independientes que conllevan alguna forma de intercambio o circulación de información sobre cuestiones relativas a una variedad de elementos de la gestión empresarial (Powell y Grodal, 2005; Sammarra y Biggiero, 2008), es un área de creciente interés tanto en el ámbito empresarial como en el académico (Gulati y Gargiulo, 1999; Mizruchi y Galaskiewicz, 1993; Uzzi, 1997). Durante los últimos años se han llevado a cabo diversos estudios de investigación que confirman la importancia que dichas redes de colaboración inter-organizacional juegan en la difusión de innovaciones (Powell y Grodal, 2005), la transferencia tecnológica (Chesbrough, 2003; Gulati y Gargiulo, 1999) o en ciertos resultados empresariales tales como la capacidad de innovar (por ejemplo, ver el número especial dedicado a las redes inter-organizacionales publicado en el Journal of Management Studies en 2008). A través de estos contactos de red las empresas están más abiertas a aprender de la experiencia de otras empresas en una variedad de situaciones, tales como el lanzamiento de proyectos experimentales, la apertura de nuevos mercados o la comprensión de las ventajas y dificultades encontradas en la implementación de nuevas estrategias y prácticas organizativas (Beckman y Haunschild, 2002; Martin-Rios 2012; Martin-Rios y Erhardt, 2007; Powell y col., 2006). Cabe, por tanto, esperar que las empresas formulen objetivos instrumentales respecto a su participación en este tipo de relaciones, en la forma de búsqueda de beneficios derivados del intercambio de conocimiento con otras empresas participantes.

De ello se deriva un argumento que es central para este estudio y la base de nuestra investigación: Las empresas tienen acceso a nuevas fuentes de conocimiento por razón de su pertenencia a una red informal y al desarrollo de relaciones más o menos profundas de intercambio con otras empresas de dicha red.

\section{Tipología de conocimiento}

Dado que el conocimiento es un recurso central para el éxito de las organizaciones (Nonaka y Takeuchi, 1999), la investigación académica ha realizado un ingente esfuerzo para desentrañar el contenido del conocimiento que se transfiere a través 
de las redes inter-organizacionales. Algunos estudios señalan que el contenido del conocimiento que se difunde en las redes inter-organizacionales es complejo y se halla altamente relacionado con las relaciones entre los diversos miembros de la red (Granovetter, 1985; Powell y col., 1996; Uzzi, 1997; Tsai, 2002).

La literatura ha desarrollado varias categorizaciones del conocimiento, incluyendo la distinción popular entre conocimiento explícito o información y conocimiento tácito o know-how (Polanyi 1966; Dyer y Nobeoka, 2000). Investigaciones recientes sobre el flujo de conocimiento (Collison y Gregson, 2003; Sammarra y Biggiero, 2008; Simonin 1999) clasifican las diversas dimensiones de conocimiento de acuerdo a su contenido y el impacto en los resultados de la organización, resultando tres prototipos:

- Conocimiento tecnológico: hace referencia a la adquisición, modificación, desarrollo, apropiación y difusión de tecnología, producción e I+D+i.

- Conocimiento de mercado o de producto/servicio: se refiere fundamentalmente a las estrategias de venta y marketing, la comercialización y los conocimientos específicos del mercado y el sector industrial (por ejemplo, la capacidad de penetrar en mercados internacionales, la capacidad de la empresa de comprender el mercado, etc.) de aquellos productos o servicios ofertados.

- Conocimiento sobre gestión empresarial: referidos a las capacidades organizativas y administrativas (por ejemplo, las capacidades para gestionar los diversos procesos administrativos de la empresa como la gestión de recursos humanos, calidad total, finanzas o logística).

Esta categorización del conocimiento es importante para el estudio de las redes informales de conocimiento entre organizaciones ya que permite a los investigadores tener en cuenta una amplia variedad de conocimientos relacionados con diversos beneficios para las empresas. En definitiva, se deriva que, a través de la creación de lazos empresariales, se establecen canales informales por donde circula información y conocimiento pertinentes para las empresas en áreas tales como las tecnología, de mercado o producto/servicio y, finalmente, de gestión empresarial. En concreto:

H1. La participación en redes informales favorece el intercambio de múltiples formas de conocimiento en áreas de importancia estratégica para las empresas. 


\section{Las características estructurales y relacionales de las redes informales}

Según la teoría de las redes sociales (Granovetter, 1985; Wasserman y Faust, 1994), las propiedades estructurales de las redes definen la naturaleza de localización, determinan las configuraciones de la red y las dinámicas de intercambio de conocimiento. Dichos aspectos se recogen en conceptos como la posición de la red (centralización) o la fuerza de la red (densidad).

Diversos estudios han mostrado que, en las redes informales, estas configuraciones estructurales son más abiertas (es decir, existe una mayor variabilidad en el número de empresas que ocupan posiciones centrales) y densas que en las redes formales (Dahl y Pedersen, 2004; Lissoni, 2001). No obstante, estas investigaciones no han examinado explícitamente las configuraciones estructurales de las redes informales de varios tipos de conocimiento, por lo que creemos que resultarán diferentes configuraciones de red en línea con el tipo de conocimientos intercambiados. De este modo, nuestra segunda hipótesis es la siguiente:

H2. Existe una relación positiva entre los factores estructurales de centralidad $y$ densidad $y$ el tipo de conocimiento que se intercambia en las redes inter-empresariales informales.

El objetivo de carácter más intangible de las redes informales hace referencia al aprendizaje y el intercambio de conocimiento (Crossan y Inkpen, 1994; Hamel, 1991; Kogut, 1988; Parkhe, 1993). Subyacente a este objetivo está la expectativa de que al transferir conocimiento se va a recibir algo a cambio. Desde esta lógica, cuando el intercambio de conocimiento conlleva el riesgo de intercambiar información de carácter estratégico, las empresas establecen acuerdos formales, debido a la limitada eficacia de los mecanismos formales en el ámbito colaborativo e informal. Pero este no es el caso en las redes informales que no requieren contratos formales para su intercambio (Appleyard 1996). En este caso, las empresas establecen relaciones altamente recíprocas para el intercambio de información. Cuestiones como la similitud en el tipo de negocio, la existencia de clientes o suministradores comunes, o factores geográficos, culturales o institucionales favorecen que empresas sin contactos comerciales formales entren en contacto unas con otras (Powell y col., 1996). Se sugiere la siguiente hipótesis sobre la relación entre el nivel de reciprocidad y el tipo de conocimiento, que se asume de manera implícita en la discusión anterior:

H3. La especificidad del tipo de conocimiento que se comparte se asocia positivamente con el nivel de reciprocidad en las redes inter-empresariales informales. 


\section{Metodología}

La teoría de redes basa su análisis en la existencia de estructuras sociales que desarrollan lazos de interdependencia entre sí. Para estudiar dichas interdependencias se utiliza el análisis de redes sociales (ARS) bien sobre redes completas o bien sobre redes que contienen las relaciones personales especificadas por determinados miembros de un grupo (conocidas como redes egocéntricas) y que exploran los lazos que los individuos entrevistados afirman tener. Uno de los principales retos del estudio de redes sociales es el poder especificar de forma adecuada los límites de las redes que se pretenden analizar (Wasserman y Faust, 1994). Por ese motivo, para el propósito de este estudio, hemos elegido analizar una red completa de empresas. Dicha red fue definida no por la existencia de relaciones formales contractuales (por ejemplo, la existencia de alianzas empresariales estratégicas o relaciones de dependencia contractual) sino por la existencia de relaciones informales y de afiliación a una comunidad de empresas alrededor de un parque científico y tecnológico

Así, este estudio de redes completas describe la estructura comprehensiva de las relaciones que se dan en un sistema social definido, como se puede afirmar que lo es un parque científico y tecnológico en dónde ya están establecidos los límites de la población a estudiar. Mediante el análisis estructural y relacional de las redes sociales se analizan las diversas formas en las que los miembros del sistema (las empresas del Parque) se encuentran conectados directa o indirectamente con el fin de colaborar y compartir conocimiento. En este caso, los nodos de las redes resultantes son las empresas y la existencia de intercambio entre los directivos de una empresa con personas de otra empresa del Parque, es empleada como señal de la existencia de un lazo entre ambas.

A continuación se ofrece una breve descripción del Parque, seguida por una descripción detallada del procedimiento de recogida de datos, el análisis llevado a cabo y la estrategia analítica seguida en este estudio.

\section{El Parque científico y tecnológico}

El parque científico donde se ha llevado a cabo el trabajo de campo de este estudio es el Parque Científico y Tecnológico de Leganés el cual se encuentra situado en el área metropolitana de Madrid. En el año 2000, la Universidad Carlos III de Madrid junto a otros organismos públicos promovió la creación del Parque Científico de Leganés con la doble finalidad de, por un lado, proveer servicios de asesoría a spin- 
offs y favorecer la consolidación de empresas surgidas de la incubadora de ideas y de, por otro lado, proveer servicios inmobiliarios y de asistencia administrativa a empresas consolidadas de alta tecnología. El principal objetivo del Parque de Leganés es crear las condiciones para que se produzcan sinergias que favorezcan la transferencia de conocimiento entre organizaciones y un mayor dinamismo e innovación (productiva y de gestión). El Parque de Leganés es un ejemplo de parque científico universitario que combina la incubación de proyectos empresariales y da apoyo institucional tanto a empresas independientes de reciente creación como aquellas con una trayectoria empresarial consolidada.

En el parque conviven cuatro entidades (60 organizaciones en total) con pesos específicos variables. Asimismo, cada entidad mantiene una proximidad variable a la oficina central de gestión del campus. El grupo con mayor peso relativo dentro del parque, con un total de 38 empresas, es el conformado por las empresas establecidas y las start-ups independientes. La incubadora de empresas integra los proyectos e ideas de negocio bajo la supervisión de la universidad (un total de cinco en el momento de realizar el trabajo de campo) y 12 spin-offs universitarios y business ventures (empresas creadas a partir de una actividad realizada en otra empresa o en una universidad, en cuyo caso se denomina spin-off académico). Finalmente, en el parque hay ubicados un total de seis centros de investigación y desarrollo avanzados, que incluyen institutos de investigación financiados con fondos públicos e institutos y consorcios auspiciados por la universidad (por ejemplo, el Centro de Integración de Sistemas Aeroespaciales EADS-UC3M).

Para el propósito de este trabajo, los centros de investigación y los proyectos de empresas en la incubadora todavía sin una idea clara de negocio fueron excluidos del estudio. La red de empresas que componen el parque está compuesta por 51 nodos (incluyendo la Oficina Tecnológica perteneciente a la universidad).

\section{Recogida y análisis de datos}

Con el fin de estudiar los procesos de intercambio de conocimiento dentro de las redes inter-organizacionales de relaciones informales o colaborativas se llevó a cabo un trabajo empírico mediante la metodología estructural o de redes sociales. Para ello, se elaboró un cuestionario que permite asignar el intercambio de conocimiento entre empresas (a través de las respuestas ofrecidas por las personas responsables de establecer y mantener relaciones con otras empresas). En dicho cuestionario se solicitó a los encuestados que señalaran con qué empresas mantuvieron contacto de una lista completa de todas las empresas pertenecientes 
al Parque. A través de la formulación de las preguntas en el cuestionario es posible revelar la existencia de una red de colaboración informal entre empresas (mediante las respuestas ofrecidas a la siguiente pregunta: "¿De entre las empresas del listado, podría indicarnos con cuáles de ellas y en qué proporción ha mantenido relaciones de colaboración informal en ámbitos relacionados con el intercambio de conocimiento?") y de diversas redes de intercambio de diversos tipos de conocimiento de relevancia para las empresas. Las preguntas sobre los tipos de conocimiento se recogen en la Tabla 1.

Tabla 1. Preguntas sobre intercambio de conocimiento incluidas en el cuestionario.

\begin{tabular}{|c|c|}
\hline \multicolumn{2}{|c|}{$\begin{array}{l}\text { De las empresas en el listado, cite aquellas con las que, en los últimos seis meses, su } \\
\text { empresa ha establecido algún tipo de intercambio de información no mediado por } \\
\text { vinculación contractual }\end{array}$} \\
\hline Tipo de conocimiento & Ejemplos \\
\hline $\begin{array}{l}\text { ¿De entre las empresas del listado, } \\
\text { podría indicarnos con cuáles de } \\
\text { ellas ha intercambiado información } \\
\text { relativa a aspectos relacionados } \\
\text { con la tecnología en los últimos } \\
\text { seis meses? }\end{array}$ & 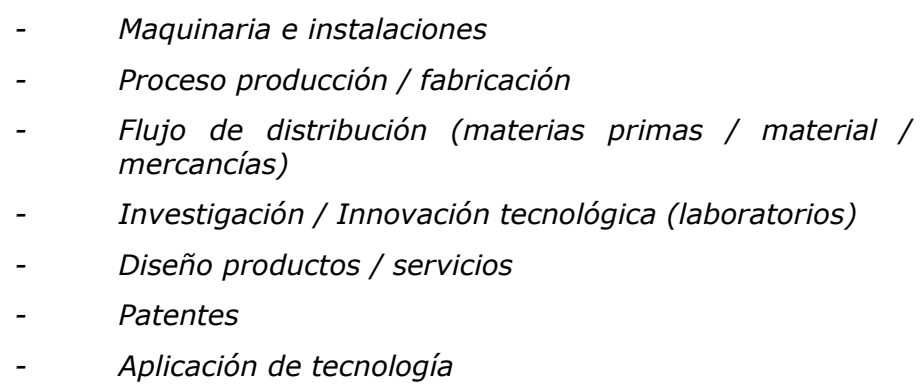 \\
\hline $\begin{array}{l}\text { ¿De entre las empresas del listado, } \\
\text { podría indicarnos con cuáles de } \\
\text { ellas ha intercambiado información } \\
\text { relativa a aspectos relacionados } \\
\text { con los productos, servicios y } \\
\text { mercado en los últimos seis } \\
\text { meses? }\end{array}$ & $\begin{array}{ll}\text { - } & \text { Planificación comercial y de mercado } \\
\text { - } & \text { Marketing } \\
\text { - } & \text { Previsión de ventas } \\
\text { - } & \text { Análisis de precios } \\
\text { - } & \text { Promoción y publicidad de productos } \\
\text { - } & \text { Prospección de nuevos mercados } \\
\text { - } & \text { Relación con clientes }\end{array}$ \\
\hline $\begin{array}{l}\text { ¿ De entre las empresas del } \\
\text { listado, podría indicarnos con } \\
\text { cuáles de ellas ha intercambiado } \\
\text { información relativa a aspectos } \\
\text { relacionados con la gestión } \\
\text { organizativa y administrativa en } \\
\text { los últimos seis meses? }\end{array}$ & $\begin{array}{ll}\text { - } & \text { Gestión de personal } \\
\text { - } & \text { Gestión de la calidad total } \\
\text { - } & \text { Gestión de proyectos } \\
\text { - } & \text { Gestión financiera } \\
\text { - } & \text { Gestión de la innovación } \\
\text { - } & \text { Gestión de producción }\end{array}$ \\
\hline
\end{tabular}

La red de empresas que forman el parque científico elegido para el trabajo de campo está compuesta por 50 empresas y la Oficina Tecnológica de la propia universidad en el Parque. El trabajo de campo tuvo una duración total de 8 semanas y se realizó en dos fases. En primer lugar, y tras el envío de una carta de presentación, se concertó una visita con el director/gerente de cada empresa del parque para que todas ellas tuvieran un $100 \%$ de probabilidad de ser seleccionada. 
Después de explicar el objetivo general del estudio, se solicitó una entrevista con el informante que más contactos realizaba con otras organizaciones como representante de su empresa (Kumar y col., 1993), es decir, aquellos que mejor pudieron evaluar qué y cómo se intercambia el conocimiento de su empresa con otras del parque. En un segundo momento, se realizaron varios intentos de contacto telefónico con aquellas empresas con las que no fue posible establecer un contacto directo. En total, se administró el cuestionario a un total de 41 empresas, representando una tasa de respuesta del $80.4 \%$. Las diez empresas restantes o bien declinaron participar o bien no fue posible establecer contacto.

Las empresas de las que no se obtuvo información corresponden a pequeñas entidades con un peso relativamente marginal en la red de colaboración. Cinco de ellas eran pequeñas spin-offs con menos de 10 empleados; el resto corresponden a pequeñas empresas de servicios auxiliares. Se computaron las nominaciones realizadas por el resto de empresas para reconstruir en la medida de lo posible la estructura de las interacciones, como se muestra en los sociogramas obtenidos. Diversos expertos en la metodología de redes han señalado que las técnicas de reconstrucción (imputar los vínculos señalados por los actores que sí participaron sobre sus relaciones con los casos perdidos) es el mejor método de imputación y arroja mejores resultados que simplemente ignorar la estructura de relaciones de los casos perdidos (Gabbay and Zuckerman, 1998; Huisman, 2009; Stork and Richards, 1992). Por ello, se realizó una imputación mediante reconstrucción de los valores perdidos, $\mathrm{Xij}$, con el fin de reemplazarlos con el valor observado de los nodos, $\mathrm{Xij}$, con los que están conectados estos actores: Xmisij = Xji. (Huisman, 2009).

\section{Análisis de datos}

La red de datos se procesó a través del paquete de software UCINET (Borgatti, Everet y Freeman, 2002) y los grafos o "sociogramas" presentados en la sección siguiente se desarrollaron utilizando la utilidad NetDraw (Borgatti, 2007). Los sociogramas representan la red como una serie de nodos. En las redes analizadas, los lazos son direccionales, con una punta de flecha que indica el sentido de la relación de colaboración. Los nodos con el mayor número de vínculos con los demás se sitúan generalmente en el centro de la red y son conocidos como nodos focales.

Se calcularon los índices de la red, midiendo la intensidad y la generalización de la transferencia de conocimientos a través de vínculos externos. Entre los diversos indicadores estructurales de análisis de redes se analizaron diversas medidas de 
centralización de la red: centralidad de grado normalizada, índice de centralización de Freeman, centralización de intermediación y centralización de cercanía. Asimismo, se calcularon la densidad y reciprocidad de las redes informales de colaboración y de intercambio de conocimiento. En la Tabla 2 se detallan las definiciones y formulación de los indicadores utilizados.

Tabla 2. Indicadores de red utilizados.

\begin{tabular}{|c|c|c|}
\hline Indicador & Concepto & Formulación \\
\hline $\begin{array}{l}\text { Centralidad de grado } \\
\text { (normalizada) }\end{array}$ & $\begin{array}{l}\text { Media del número de nodos con los que } \\
\text { están conectados cada actor. El valor de H } \\
\text { se maximiza cuando el grafo incluye un } \\
\text { nodo focal al cual se conectan todos los } \\
\text { demás. }\end{array}$ & $C_{D(G)}=\frac{\sum_{i=1}^{N}\left[C_{D}(v *)-C_{D}\left(v_{i}\right)\right]}{H}$ \\
\hline $\begin{array}{l}\text { Índice de } \\
\text { centralización }\end{array}$ & $\begin{array}{l}\text { Grado en que una red se organiza alrededor } \\
\text { de un punto o zona central (Freeman, } \\
1979 \text { ). Se computa dividiendo la suma de } \\
\text { las diferencias de centralidad del nodo focal } \\
\text { con el resto de nodos por el máximo } \\
\text { número posible de vínculos en la red } \\
\text { (Borgatti and Everett, 1997). }\end{array}$ & $C_{D}=\frac{\sum_{i=1}^{N}\left(c_{\max }-c_{i}\right)}{\max \left\{\sum_{i=1}^{N}\left(c_{\max }-c_{i}\right)\right\}}$ \\
\hline $\begin{array}{l}\text { Centralización de } \\
\text { intermediación }\end{array}$ & $\begin{array}{l}\text { Medida de la dependencia que los actores } \\
\text { tienen de los nodos focales para realizar sus } \\
\text { contactos. CB indica la varianza en las } \\
\text { centralidades de intermediación de todos los } \\
\text { nodos, donde C_B ( } \mathrm{n}^{\wedge *} \text { ) indica la } \\
\text { intermediación mayor existente entre el } \\
\text { conjunto de actores (Wasserman and Faust, } \\
\text { 1994) }\end{array}$ & $C_{B}=\frac{2 \sum \frac{g}{i=1}\left[C_{B}\left(n^{*}\right)-C_{B}\left(n_{i}\right)\right]}{\left[(g-1)^{2}(g-2)\right]}$ \\
\hline $\begin{array}{l}\text { Centralización de } \\
\text { cercanía }\end{array}$ & $\begin{array}{l}\text { Medida radial de longitud que calcula el } \\
\text { promedio de las distancias geodésicas más } \\
\text { cortas de los actores (CC) hacia todos los } \\
\text { demás (Wasserman and Faust, 1994). }\end{array}$ & $\mathrm{C}_{\mathrm{c}}=\sum_{x \in V-C} d f(x, C)$ \\
\hline Densidad & $\begin{array}{l}\text { Medida del número de vínculos existentes (i } \\
\text { a j) en relación al número de vínculos } \\
\text { posibles, calculados como } n(n-1) \text { (Scott } \\
\text { 1991). }\end{array}$ & $\Delta=\frac{\sum_{i=1}^{N} \sum_{j=1}^{N} x_{i j}}{n(n-1)}$ \\
\hline Reciprocidad & $\begin{array}{l}\text { El número de vínculos que implican } \\
\text { interacciones diádicas mutuas sobre el total } \\
\text { de vínculos (Wasserman and Faust 1994), } \\
\text { donde M es el número total de vínculos } \\
\text { diádicos mutuos y A las relaciones } \\
\text { asimétricas en la red. }\end{array}$ & $R=\frac{2 M}{2 M+A}$ \\
\hline
\end{tabular}

\section{Características de las empresas analizadas}

De acuerdo con la clasificación CNAE-2009, todas las empresas analizadas operan en sectores de tecnología alta, bien en sectores manufactureros de tecnología alta y media-alta (industria farmacéutica, maquinaria de oficina y material informático, componentes electrónicos, aparatos de radio, TV y comunicaciones, instrumentos médicos, de precisión, óptica y relojería, construcción aeronáutica y espacial, industria química, maquinaria y equipos mecánicos, aparatos eléctricos, industria automóvil) o en servicios de alta tecnología 0 de punta (correos y 
telecomunicaciones, actividades informáticas o investigación y desarrollo). La antigüedad media de las empresas es de 11,7 años. El noventa por ciento de las empresas son PYMEs, mientras que el diez por ciento restantes son empresas de más de 200 empleados con facturaciones anuales superiores a los 15 millones de euros. Las características de la muestra son las siguientes:

Tabla 3. Características de las empresas ${ }^{3}$.

\begin{tabular}{|c|c|c|c|c|c|}
\hline \multicolumn{2}{|r|}{ Variable } & \multicolumn{4}{|c|}{ Distribución de frecuencias $(\mathrm{N}=51)$} \\
\hline 1. & Antigüedad (años) & Menos de 10 & 11-20 & 21-30 & Total \\
\hline & $v . a^{\wedge}(\%)$ & $35(68,6 \%)$ & $11(21,6 \%)$ & $5(9,8 \%)$ & $51(100 \%)$ \\
\hline 2. & Actividad* & MAT & SAT & Otros & Total \\
\hline & v.a (\%) & $17(33,3 \%)$ & $33(64,7 \%)$ & $1(1,9 \%)$ & $51(100 \%)$ \\
\hline 3. & Empleados 09 & Menos de 25 & $25-200$ & Más de 201 & Total \\
\hline & v.a (\%) & $15(35,7 \%)$ & $23(54,8 \%)$ & $4(9,5 \%)$ & $42(100 \%)^{+}$ \\
\hline 4. & Facturación 09 (mill $€$ ) & Menos de 1 & 1-15 & Más de 15 & Total \\
\hline & v.a (\%) & $10(23,8 \%)$ & $22(52,4 \%)$ & $10(23,8 \%)$ & $42(100 \%)^{+}$ \\
\hline 5. & Mercado & Nacional & Internacional & Total & \\
\hline & v.a (\%) & $24(48 \%)$ & $26(52 \%)$ & $50(100 \%)^{!}$ & \\
\hline 6. & Origen negocio & Independiente & Spin-off & $\begin{array}{l}\text { Subsidiaria } \\
\text { multinacional }\end{array}$ & Total \\
\hline & v.a (\%) & $38(74,5 \%)$ & $11(21,6 \%)$ & $2(3,9 \%)$ & $51(100 \%)$ \\
\hline
\end{tabular}

\section{Resultados}

Con respecto a la estructura reticular de las relaciones de intercambio de conocimiento, como se ve en el Gráfico 1, la red informal de colaboración entre empresas del Parque se encuentra bien consolidada. El número de empresas aisladas respecto al resto es muy reducido. Únicamente cuatro empresas están completamente desconectadas del resto. Se aprecia, además, que son varias las empresas focales, altamente mencionadas, y que el número de lazos de colaboración entre empresas es considerable. En los sociogramas la fortaleza de los vínculos viene representada por el grosor de las líneas. Se observa que los vínculos fuertes no se producen exclusivamente entre las empresas con mayor centralidad (caracterizado por el tamaño de los nodos) si no que existen relaciones fuertes también entre empresas relativamente menos centrales en la red.

\footnotetext{
* MAT: manufactura alta tecnología; SAT: servicios alta tecnología

v.a.: valor absoluto

+9 casos perdidos

! 1 caso perdido
} 
Cabe destacar la centralidad relativa de la oficina tecnológica del Parque (39) en todas las redes de conocimiento. A la luz del análisis de la centralizada de intermediación de los nodos (que por cuestiones de espacio no se incluye en este estudio), la oficina actúa de bróker o intermediario en los intercambios de conocimiento siendo un dinamizador de dichos intercambios. Esto puede ser debido en parte al hecho de que la oficina, además de gestionar la incubadora de empresas, tiene como uno de sus objetivos el favorecer la creación de lazos de intercambio entre las diversas empresas. Estos resultados son similares a los obtenidos en otros estudios realizados en parques tecnológicos (Beise y Stahl, 1999; Löfsten y Lindelof, 2002). Sin embargo, cabe destacar que, al no ejercer control sobre recursos claves (por ejemplo, recursos financieros o personal clave que puede ser crítico para el desempeño de otras empresas), se puede afirmar que no una existe dependencia de recursos por parte de las empresas hacia la oficina. Quizá por ello, mantiene una posición de liderazgo incluso en la red de colaboración informal.

Gráfico 1. Red de colaboración informal (centralidad de grado para tamaño de nodo)

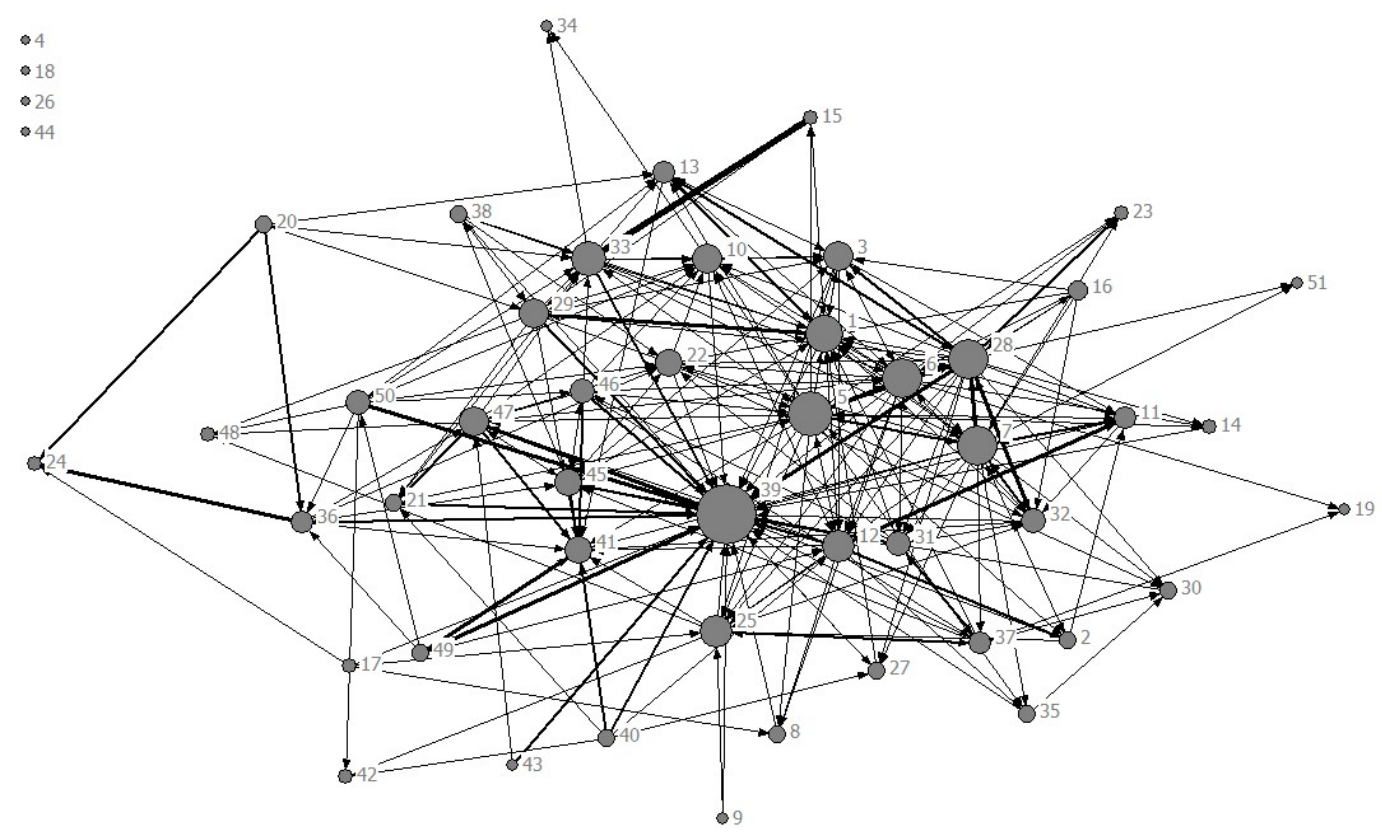

Para testar la hipótesis 1 que propone la existencia de beneficios para las empresas participantes en redes informales derivados del acceso a nuevas fuentes de conocimiento en una variedad de dominios, se realizó el análisis del contenido del conocimiento intercambiado entre las empresas. El Gráfico 2 muestra la red de empresas que mantienen intercambios de conocimiento tecnológico. Esta red 
confirma la alta participación de las empresas y el escaso aislamiento relativo (únicamente seis empresas se encuentran aisladas). De nuevo, la oficina del Parque (39) ha desarrollado vínculos fuertes con múltiples empresas en la red y sirve de bróker en la transferencia de conocimiento entre los diferentes grupos. De deduce, además, que diversas empresas participan activamente en la obtención e el intercambio de conocimiento tecnológico, lo cual puede atribuirse a la propia naturaleza de las empresas participantes: todas ellas realizan su actividad en áreas con una demanda alta de competencias relacionadas con la tecnología.

Gráfico 2. Red de intercambio de conocimiento tecnológico (centralidad de grado para tamaño de nodo)

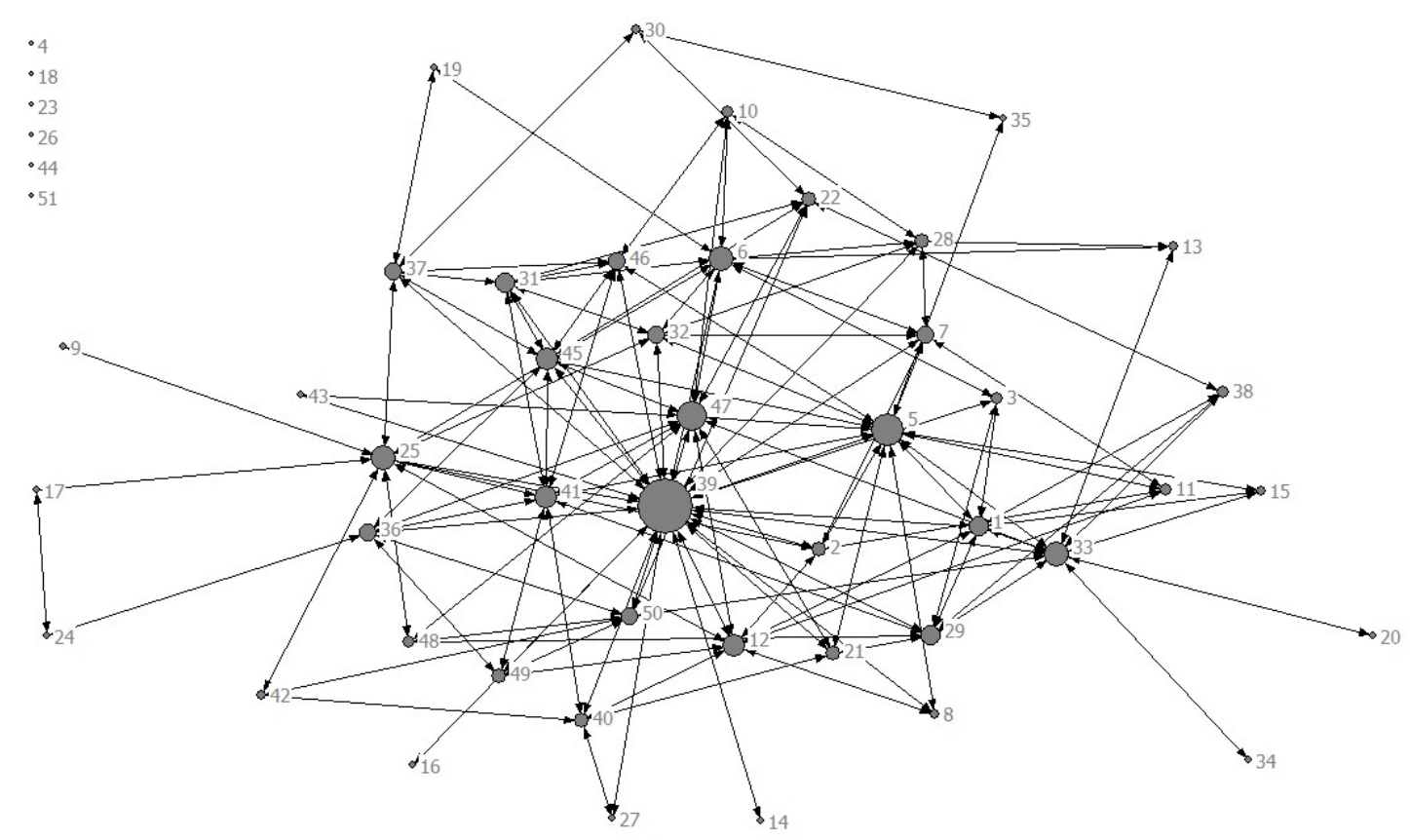

Respecto al intercambio de conocimiento de mercado, el Gráfico 3 muestra que, en comparación la red de conocimiento tecnológico, las empresas establecen menores intercambios y dichos intercambios tienen un carácter más esporádico y horizontal (menos centralizado, tal y como lo indica el índice de centralización que se analiza más abajo). 
Gráfico 3. Red de intercambio de conocimiento de mercado (centralidad de grado para tamaño de nodo)

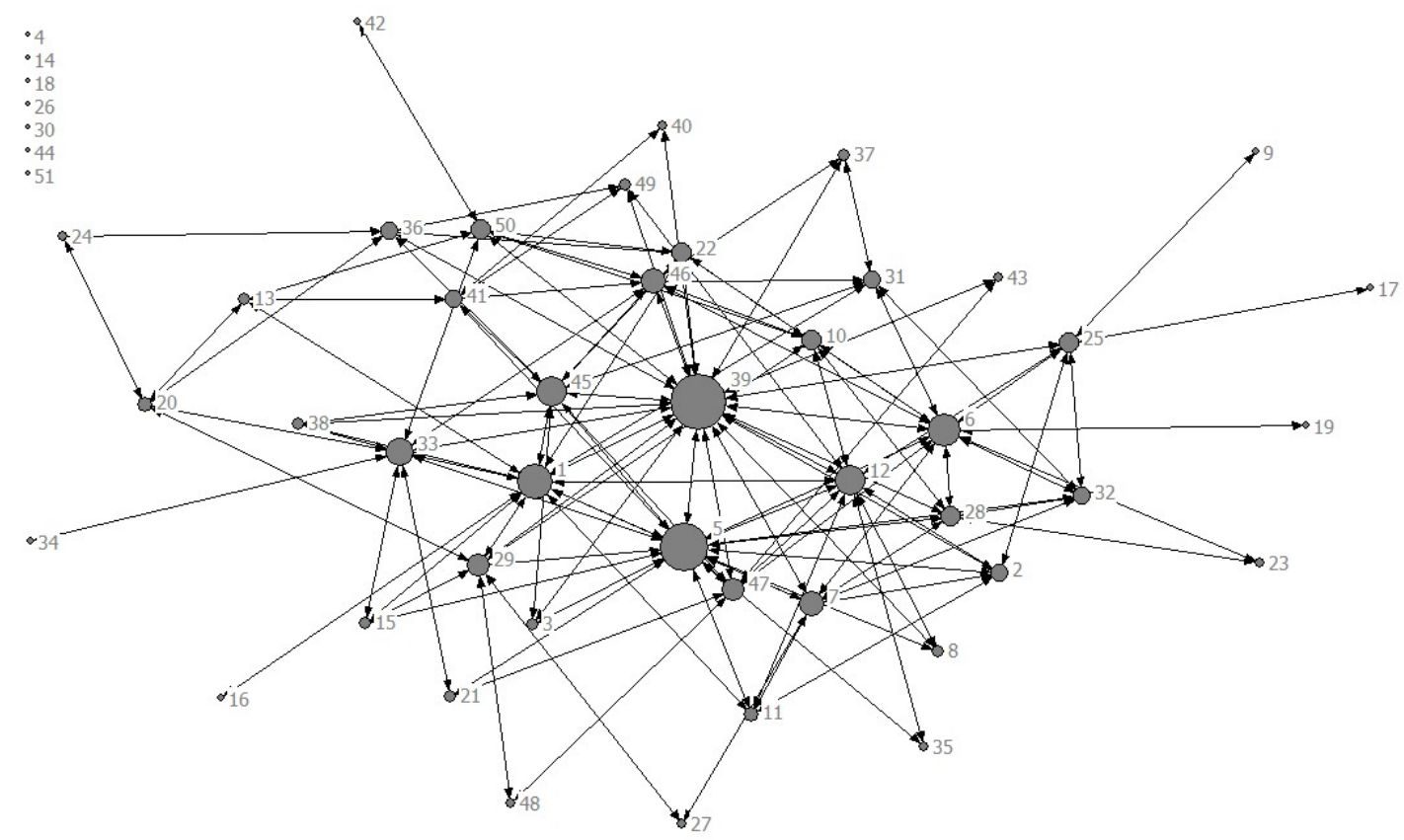

Finalmente, se ha elaborado la red de intercambio de conocimiento en materias relativas a la gestión empresarial. La existencia tanto de una red de conocimiento de mercado como de una red de conocimiento sobre aspectos de organización y gestión empresarial surgidas de las relaciones informales y colaborativas entre empresas independientes constituye una aportación importante al estudio de este tipo de redes.

Gráfico 4. Red de intercambio de conocimiento de gestión (centralidad de grado para tamaño de nodo)

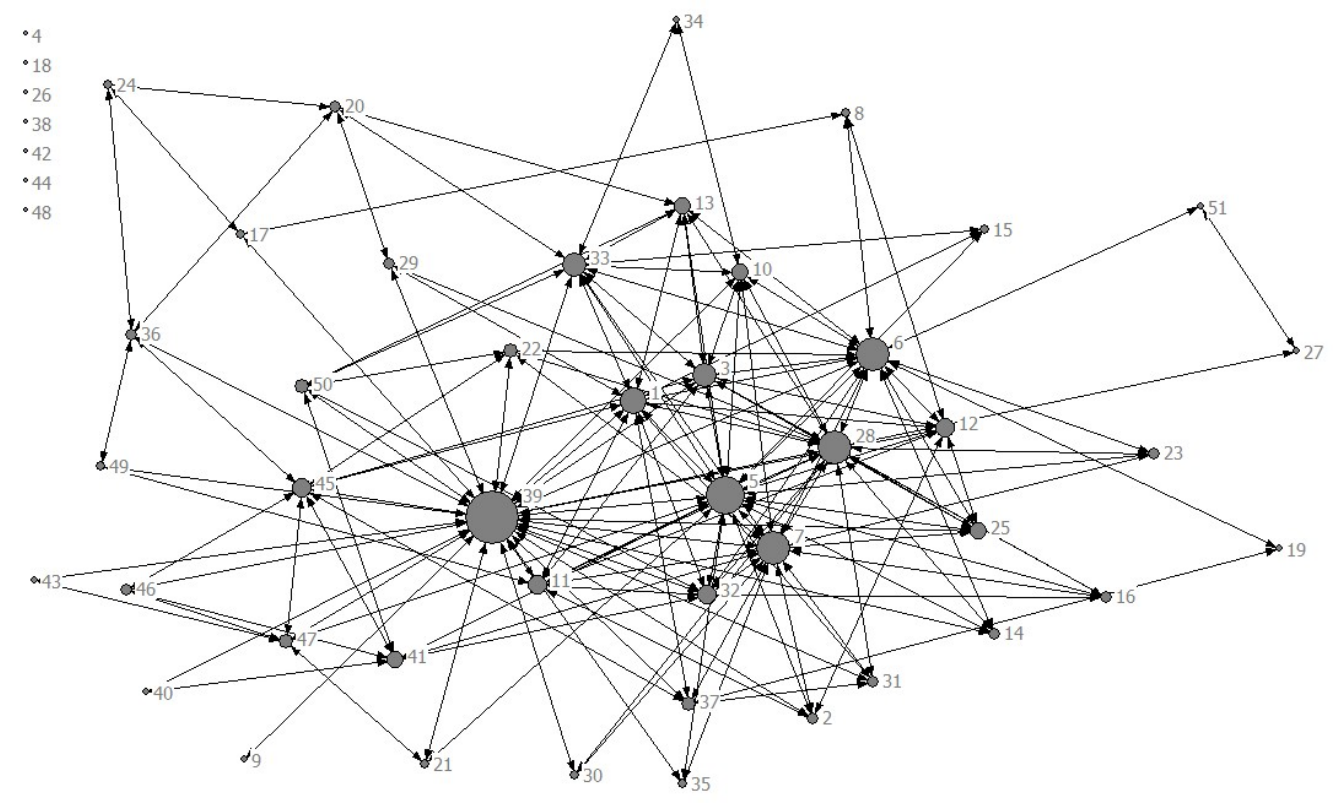


Estos resultados dan validez a la primera hipótesis y permiten reflexionar sobre el papel de las redes informales de intercambio de conocimiento. Si bien intuitivamente cabe esperar que este tipo de redes sean multidimensionales por naturaleza, existen muy pocas investigaciones que hayan explorado empíricamente dicha eventualidad. Las redes aquí descritas a través de su grafo permiten poner de manifiesto la existencia de una heterogeneidad respecto al contenido de la red.

Con el fin de testar la validez de la segunda hipótesis que plantea la existencia de una relación positiva entre las dimensiones estructurales y el tipo de conocimiento que se intercambia, se han analizado los factores estructurales de centralidad y densidad de las redes de intercambio de conocimiento entre empresas. Para tal fin se han calculado diversos indicadores de centralización y densidad. Estos indicadores permiten analizar los niveles de participación de las empresas en las redes de intercambio y el grado de jerarquización de cada una de las redes. Los indicadores de cada elemento se detallan en la Tabla 4.

Tabla 3. Descriptores y principales resultados estructurales de las redes de conocimiento.

\begin{tabular}{|c|c|c|c|c|}
\hline & $\begin{array}{l}\text { Red informal } \\
\text { de } \\
\text { colaboración }\end{array}$ & $\begin{array}{l}\text { Red de } \\
\text { intercambio } \\
\text { de } \\
\text { conocimiento } \\
\text { tecnológico }\end{array}$ & $\begin{array}{l}\text { Red de } \\
\text { intercambio de } \\
\text { conocimiento } \\
\text { de mercado }\end{array}$ & $\begin{array}{l}\text { Red de } \\
\text { intercambio de } \\
\text { conocimiento } \\
\text { de gestión }\end{array}$ \\
\hline \multicolumn{5}{|l|}{$\begin{array}{l}\text { Características estructurales y } \\
\text { relacionales de las redes }\end{array}$} \\
\hline Valor de las relaciones & Ordinal & Binario $(0-1)$ & Binario $(0-1)$ & Binario $(0-1)$ \\
\hline Tamaño (numero de nodos) & 51 & 51 & 51 & 51 \\
\hline Nodos aislados & $4 / 51=7,8 \%$ & $6 / 51=11,8 \%$ & $7 / 51=13,7 \%$ & $7 / 51=13,7 \%$ \\
\hline $\begin{array}{l}\text { Centralidad de grado } \\
\text { normalizada }\end{array}$ & 9,830 & 5,255 & 5,059 & 6,196 \\
\hline Índice de centralización (\%) & 54,00 & 45,27 & 37,35 & 51,63 \\
\hline $\begin{array}{l}\text { Centralización de } \\
\text { intermediación (\%) }\end{array}$ & 57,15 & 54,64 & 48,22 & 58,39 \\
\hline $\begin{array}{l}\text { Centralización de cercanía } \\
(\%)\end{array}$ & 8,94 & 11,75 & 8,91 & 9,27 \\
\hline Densidad & 0,1812 & 0,1051 & 0,1012 & 0,1239 \\
\hline $\begin{array}{l}\text { Total conocimiento } \\
\text { intercambiado (suma de } \\
\text { grados) }\end{array}$ & 462,00 & 316,00 & 258,00 & 316,00 \\
\hline Porcentaje de lazos recíprocos & $16,45 \%$ & $18,69 \%$ & $11,32 \%$ & $17,32 \%$ \\
\hline
\end{tabular}


En primer lugar, cabe destacar la existencia de unas redes relativamente densas de intercambio de conocimientos. La densidad de la red (el nivel de participación en la red) presenta unos valores similares en las tres redes de conocimiento $(0,105$, 0,101 and 0,124 respectivamente). Se puede afirmar que las redes que exhiben mayores niveles de densidad están más interconectadas que aquellas otras redes con baja densidad, facilitando mayores vínculos para el intercambio de conocimiento. Atendiendo a los niveles de densidad, el intercambio de conocimientos de mercado y tecnológico es menor que el relativo a conocimiento organizativo. Si bien las densidades no pueden compararse entre redes de diferente tamaño y actores (Scott, 1991), intuitivamente estos resultados contrastan con los obtenidos en estudios anteriores donde se reporta la menor recurrencia de intercambios informales de conocimiento (Boschma and ter Wal, 2007; Rank y col., 2006).

El índice de centralización es mayor en la red del conocimiento de gestión y menor en la red de conocimiento tecnológico, lo que indica que los flujos de conocimientos tecnológicos están menos centralizados en torno a las empresas individuales. Curiosamente, la centralización de intermediación es más alta para la red de conocimiento tecnológico. En una red con una mayor centralización de intermediación es probable que existan más empresas (puentes) que conectan a grupos desconectados. Con respecto a los índices de centralización de cercanía son relativamente bajos, lo que implica niveles no muy altos de acceso a los conocimientos centralizados, siendo el acceso centralizado levemente mayor en el conocimiento de gestión $(58,4 \%)$ y en el conocimiento tecnológico $(54,6 \%)$, que para el conocimiento de mercado $(48,2 \%)$. Finalmente, los resultados obtenidos para cada red en términos de centralidad de grado normalizada son: 5,26, 5,1 y 6,2 . La diferencia entre estas medidas de centralización indica que, de media, las empresas tienen más de cinco intercambios colaborativos de conocimiento con otras empresas en la red.

En conclusión, los indicadores estructurales señalan que las empresas están más conectadas entre sí en la búsqueda del conocimiento que en el propio intercambio. Las empresas del Parque intercambian simultáneamente los tres tipos de conocimiento, a pesar de su mayor participación en redes de conocimiento tecnológico. Aunque las tres redes de intercambio prácticamente comprenden todas las empresas locales, el número real de intercambios de conocimiento es mucho más "desigual y selectivo", lo que apoya la Hipótesis 2, previamente formulada. 
Finalmente, se analiza la hipótesis tercera, la cual anticipa el papel positivo que la reciprocidad entre empresas juega a la hora de favorecer la existencia de relaciones de intercambio informal. El porcentaje de lazos recíprocos (antes de simetrizar la red) varía en las tres redes. Mientras que en la red de conocimiento tecnológico un $18,7 \%$ de los lazos son recíprocos, en el caso de las redes de conocimiento de mercado y de conocimiento de gestión es del $11,32 \%$ y $17,32 \%$, respectivamente. Cabe deducir que cuanto más relevante es el conocimiento objeto de intercambio para las empresas, mayor es la probabilidad de que éstas desarrollen vínculos de reciprocidad fuertes con los que establecer mayores niveles de confianza en el interlocutor. Estos resultados permiten corroborar la tercera hipótesis. Para estas empresas que operan en sectores con un alto contenido tecnológico, el conocimiento tecnológico es un componente esencial para su éxito y competitividad. A falta de un análisis detallado, esta correspondencia indica la posible existencia de niveles de confianza hacia determinadas empresas que van más allá de los lazos que las personas desarrollan con personas de otras empresas a título individual.

\section{Discusión}

Este artículo explora el papel de las redes informales de intercambio de conocimiento entre empresas como vehículo para el intercambio de conocimiento en diversos aspectos. La principal conclusión que se desprende del presente estudio es que la creación de redes no contractuales entre empresas favorece el desarrollo de relaciones de colaboración a través de las cuales se establecen canales para el intercambio de información, conocimiento y experiencias. A pesar de la ausencia de incentivos económicos evidentes, dichas redes de conocimiento brindan oportunidades a las empresas integrantes para obtener recursos en forma de conocimiento. En este sentido, coherente con dicha conclusión, determinadas empresas potencian y consolidan dichas relaciones de cooperación interorganizativas. La pertenencia a este tipo de redes beneficia a sus miembros en la adquisición e intercambio de formas heterogéneas de conocimiento, en concreto los diagramas estructurales evidencian la existencia de intercambios en tres áreas de conocimiento: tecnología (innovación y desarrollo, I+D+i, patentes y producción); mercado y productos (nacionales e internacionales, productos y servicios, marketing, marcas y posicionamiento); y gestión empresarial (incluyendo gestión de recursos humanos, calidad total, logística o finanzas). 
De los sociogramas obtenidos se constata que el parque analizado constituye en sí mismo una red informal de colaboración entre empresas. En general, las redes de conocimiento presentes en el parque estudiado muestran unos niveles de densidad (el grado de homogeneidad de los contactos) moderadamente altos (en comparación con los hallados en otros estudios sobre redes organizativas informales), unos niveles de centralidad (el grado de jerarquía de las relaciones) más bien bajos; y unos niveles de reciprocidad considerables. Estos rasgos estructurales y relacionales son distintivos de cada red de conocimiento.

Las empresas muestran variaciones de comportamiento en las diversas redes de conocimiento. Cuando el conocimiento intercambiado es percibido como estratégico para las empresas, éstas favorecen las relaciones con mayores niveles de reciprocidad, alrededor de ciertos nodos centrales. Así ocurre en el caso de la red de conocimiento tecnológico. Por otro lado, cuando el conocimiento es beneficial pero no percibido como esencial (como en el caso del conocimiento de mercado), las empresas establecen relaciones abiertas y esporádicas. En el caso del conocimiento de gestión, las empresas muestran mayores niveles de reciprocidad que en la red de conocimiento de mercado, pero menores niveles de centralización que en la red de conocimiento tecnológico. En este caso, cabe teorizar sobre la importancia que el conocimiento de gestión tiene en la innovación de las empresas de servicios (Evangelista y Vezzano, 2008), en comparación con las empresas de manufactura, donde las iniciativas innovadoras surgen con frecuencia de innovaciones de carácter tecnológico. Dado que el parque está compuesto por empresas de manufactura y de servicios, puede argumentarse que el conocimiento de gestión tiene una relevancia diferente para cada uno de estos dos grupos de empresas.

Estos hallazgos sugieren que las empresas dan importancia a la ampliación de su base de conocimiento mediante la incorporación de nuevos conocimientos a partir de fuentes externas. Cabe concluir, por tanto, que la participación de las empresas en las redes de colaboración e intercambio de conocimiento puede contribuir positivamente en el desarrollo de fuentes potenciales de ventaja competitiva para dichas empresas. Mediante la participación en dichas pueden obtener y combinar aquellos recursos de conocimiento que son importantes para su actividad.

\section{Limitaciones y conclusiones}

Este estudio presenta una serie de limitaciones y de oportunidades para futuras investigaciones. En primer lugar, el parque científico donde se llevó a cabo el 
trabajo de campo ha sido fundado hace relativamente poco tiempo y, por tanto, el grado de imbricación entre empresas se ve influido por la juventud del parque. Las redes inter-organizativas son todavía incipientes y, cabe esperar que las relaciones sean algo inestables. Finalmente, la existencia de una colaboración relativamente intensa entre las empresas podría atribuirse potencialmente al hecho de que la mayor parte de ellas, en su calidad de empresas de base tecnológica, operan en sectores similares y tienen un tamaño, un volumen de negocio y una orientación internacional similar, $y$, por ello, pueden compartir preocupaciones similares con respecto a cuestiones tecnológicas, de mercado o de gestión empresarial. Estas cuestiones abren oportunidades para futuras investigaciones. En primer lugar el estudio de los intercambios de conocimiento entre empresas independientes se vería favorecido por investigaciones que lleven a cabo estudios dinámicos de series temporales. Junto a ello, los resultados alcanzados podrían ser validados mediante la realización de comparativas entre diversos parques más o menos consolidados con el fin de comparar entre diversos niveles de imbricación e intercambio de conocimiento.

En conclusión, con este análisis se ha pretendido avanzar en el conocimiento de las relaciones informales y colaborativas entre empresas y su importancia en el desarrollo de redes de conocimiento. Los resultados de este estudio muestran la existencia de flujos más o menos densos de intercambio de conocimientos sobre aspectos relacionados con la tecnología, el mercado y la gestión empresarial, los cuales tienen lugar a través de redes de conocimiento no contractuales.

\section{Referencias bibliográficas}

Appleyard, M. (1996). How does knowledge flow? Inter-firm patterns in the semiconductor industry. Strategic Management Journal, 17: 137-154.

Beckman, M. C. y Haunschild, P. R. (2002). Network learning: The effects of partners' heterogeneity of experience on corporate acquisitions, Administrative Science Quarterly, 47: 92-124.

Beise, M. y Stahl, H. (1999). Public research and industrial innovations in Germany. Research Policy, 28: 397-422.

Borgatti, S.P. y Everet, M. (1997). Network analysis of two-mode data. Social Networks, 19: 243-269.

Borgatti, S., Everet, M., y Freeman, L. (2002). UCINET for Windows: Software for social network analysis. Harvard: Analytic Technologies. 
Borgatti, S.P. (2007). NetDraw: Graph visualization software. Harvard: Analytic Technologies.

Boschma, R. y ter Wal, A.L.J. (2007). Knowledge networks and innovative performance in an industrial district: The case of a footwear district in the south of Italy. Industry and Innovation, 14: 177-199.

Burt, R. S. y Minor, M. J. (1983). Applied Network Analysis: A Methodological Introduction, Beverly Hills: Sage.

Chesbrough, H. (2003). The era of open innovation. Boston: Harvard Business School Press

Colombo, M., Laursen, K., Magnusson, M., y Rossi-Lamastra, C. (2011). Organizing inter- and intra-firm networks: What is the impact on innovation performance? Industry \& Innovation, 18: 531-538.

Collinson, S. y Gregson, G. (2003). Knowledge networks for new technology-based firms: An international comparison of local entrepreneurship promotion. $R \& D$ Management, 33: 189-208.

Crossan, M.M., y Inkpen A.C. (1994). Promise and reality of learning through alliances, The International Executive, 36: 263-273.

Dahl, M.S. y Pedersen, C.Ø.R. (2004). Knowledge flows through informal contacts in industrial clusters: Myth or reality? Research Policy, 33: 1673-1686

Dyer J.H. y Nobeoka K. (2000). Creating and managing a high-performance knowledge-sharing network: The Toyota case. Strategic Management Journal, 21: 345-367.

Easterby-Smith, M., Lyles, M.A. y Tsang, E. (2008). Inter-organizational knowledge transfer: Current themes and future prospects. Journal of Management Studies, 45: 677-690.

Evangelista, R. y Vezzani A. (2010). The economic impact of technological and organizational innovations. A firm-level analysis. Research Policy, 39: 12531263.

Grandori, A. y Soda G. (1995). Inter-firm networks: Antecedents, mechanisms and forms. Organization Studies, 16: 183-214.

Granovetter, M.S. (1985). Economic action and social structure: The problem of embeddedness. American Journal of Sociology, 91: 481-510. 
Granovetter, M.S. (1992). Economic institutions as social constructions: A framework for analysis. Acta Sociologica, 35: 3-11.

Gabbay, S. y Zuckerman, E. (1998). Social capital and opportunity in corporate R\&D: The contingent effect of contact density on mobility expectations. Social Science Research, 27: 189-217.

Gulati, R. y Gargiulo, M. (1999). Where do inter-organizational networks come from? American Journal of Sociology, 104: 1398-1438.

Hagedoorn, J. (1993). Understanding the rationale of strategic technology partnering: Inter-organizational modes of cooperation and sectorial differences. Strategic Management Journal, 14: 371-385.

Hamel, G. (1991). Competition for competence and inter-partner learning within international strategic alliances. Strategic Management Journal, 12: 83-103.

Huisman, M. (2009). Imputation of missing network data: Some simple procedures. Journal of Social Structure, 10: 1-22.

Kogut, B. (1988). Joint ventures and the option to expand and acquire, Management Science, 37: 19-33.

Kumar N., Stern L.W. y Anderson J.C. (1993). Conducting inter-organizational research using key informants. Academy of Management Journal, 36: 16331651.

Liebeskind, J., Lumerman, A., Zucker L., y Brewer M. (1996). "Social Networks, Learning, and Flexibility: Sourcing Scientific Knowledge in New Biotechnology Firms". Organization Science, 7: 428-443.

Lissoni, F. (2001). Knowledge codification and the geography of innovation: The case of Brescia mechanical cluster. Research Policy, 30: 1479-1500.

Löfsten H. y Lindelöf, P. (2002). Science parks and the growth of new technologybased firms-academic-industry links, innovation and markets. Research Policy, 31: 859-876.

Mizruchi, M. y Galaskiewicz, J. (1993). Networks of interorganizational relations. Sociological Network and Research, 22: 46-70. Thousand Oaks, CA: Sage.

Martin-Rios, C. (2012). Why do firms seek to share human resource management knowledge? The importance of inter-firm networks. Journal of Business Research. 
Martin-Rios, C. y Erhardt, N. L. (2007). Organisational knowledge transfer through human resource management: international diffusion of managerial performance management. European Journal of International Management, 2: 170-191.

Nonaka, I. y Takeuchi, H. (1995). The Knowledge-Creating Company: How Japanese Companies Create the Dynamics of Innovation. Oxford University Press, USA.

Parkhe, A. (1993). Strategic alliance structuring: A game theoretic and transaction cost examination of interfirm cooperation. Academy of Management Journal, 36: 794-829.

Polanyi, M. (1962). Personal Knowledge: Towards a Post-critical Philosophy. Psychology Press.

Powell, W., Koput, K., y Smith-Doerr, L. (1996). "Interorganizational collaboration and the locus of innovation: Networks of learning in biotechnology". Administrative Science Quarterly, 41: 116-145.

Powell, W.W., y Grodal, S. (2005). Networks of Innovators. En J. Fagerberg, D. Mowery, y R.R. Nelson, (eds.), The Oxford Handbook of Innovation, (56-86). New York: Oxford University Press.

Rank, C., Rank O. y Wald, A. (2006). Integrated versus core-periphery structures in regional biotechnology networks. European Management Journal, 24: 73-85.

Sammarra, A., y Biggiero, L. (2008). Heterogeneity and specificity of inter-firm knowledge flows in innovation networks. Journal of Management Studies, 45: 800-829.

Scott J. (1991). Social Network Analysis: A Handbook. Thousand Oaks: Sage Pub.

Simonin, B. (1987). The importance of collaborative know-how: An empirical test of the learning organization. Academy of Management Journal, 40: 1150-1174.

Stork, D. y Richards, W. (1992). Nonrespondents in communication network studies. Group \& Organization Management, 17: 193-209.

Teece, D. (1987). The Competitive Challenge: Strategies for Industrial Innovation and Renewal. Cambridge, UK: Ballinger Publishing Company.

Tödtling F., Lehner P. y Kaufmenn A. (2009). Do different types of innovation rely on specific kinds of knowledge interactions? Technovation, 29: 59-71. 
Tsai, W. (2002). Social structure of coopetition within a multiunit organization: Coordination, competition, and intra-organizational knowledge sharing. Organization Science, 13: 179-190.

Uzzi, B. (1997). Social structure in interfirm networks: The paradox of embeddedness. Administrative Science Quarterly. 42, 35-67.

van Wijk, R., Jansen, J. y Lyles, M.A. (2008). Inter- and intra-organizational knowledge transfer: A meta-analytic review and assessment of its antecedents and consequences. Journal of Management Studies, 45: 830-853.

Wasserman S. y Faust K. (1994). Social Network Analysis: Methods and Applications. Cambridge University Press: New York.

Wellman, B. (1983). Network analysis: Some basic principles. Sociological Theory, 1: $155-200$. 